\title{
The Mechanism of Experience, Representation, and Verbal Reasoning on the Visually Impaired Individuals
}

\author{
Siti Ina Savira, Hermien Laksmiwati \\ Department of Psychology \\ Universitas Negeri Surabaya \\ Surabaya, Indonesia \\ sitisavira@unesa.ac.id
}

\author{
Wagino \\ Department of Special Education \\ Universitas Negeri Surabaya \\ Surabaya, Indonesia
}

\begin{abstract}
This study aimed to explain the mechanism of experience, representation, and verbal reasoning on people with visual impairment. The methods used in this study were focused group discussion (FGD), interview, and think-aloud. The result of this study showed that participants often used concrete versus abstract comparison to explain their difficulties in understanding and verbal reasoning; however, the term concrete often refers to something they could immediately experience, while abstract means something that requires visual information. Some concepts may be abstract, but it can be understood if it is related to their personal experience. Concrete and abstract therefore, is a way to describe the limitation of the participant's mental representation of object. It is also suggested that early experience on childhood is the primary foundation for the future object representation that determines one's ability in abstract concept formation.
\end{abstract}

Keywords-experience; verbal reasoning; visual impairment; representation

\section{INTRODUCTION}

Debates surrounding the cognitive ability of the visually impaired individuals are yet to settle [1]. Visual impairment is all type of deficit in sight [2]. One of the classic argument is whether children with visual impairment have different cognitive ability compared to their sighted counterparts, for instances in terms of intelligence and reasoning [3, 4], imagery and spatial ability [5-7], memory [8,9], and orientation and mobility [4]. This study is aimed take part in diminishing the argument and shift to a more productive way to improve the well-being of individuals with visual impairment.

Many beliefs that visual experience is important to engaging in higher-order cognitive tasks, such as reasoning [10] since it is the main channel we use to observe environment [11]. Reasoning is the ability to make inferences or conclusion from information [12]. Therefore, it often requires one to engage in abstraction, or to go beyond the concrete experience. Experience and prior knowledge are also considered to have significant affect in reasoning [10,13], because the nature of reasoning allows individual to develop their own meaning in contrast to the information they receive. This seems to be the most common foundation of arguments surrounding the discussion on reasoning ability of individuals with visual impairment; that the loss of visual experience would compromise individuals to engage in complex cognitive tasks such as reasoning. However, many research supports that the cognitive process in reasoning may involves different areas of activation in the brain than those used for visual imagery [13, 14]. Another study shows that visual impairment does not reduce the ability to process and transform mental imagery [7], which may also related to the various ways of abstraction. The argument has now shift to a more subtle area; “...the question of degree of relationship between vision and conceptualization ability" [15 p.191]. One area of research is investigating the common misconception $[16,17]$ of the visually impaired in hope to find the link between the loss of vision with reasoning and conceptual understanding of individuals with visual impairment.

Abstraction is closely associated to verbal reasoning and is assumed to be inhibited by the visual impairment [15]. In effort to understand reasoning and abstraction, studies found that the delay in verbal reasoning in the visually impaired is due to the delay in the representational play, where children with sights may explore different perspective and engage in role-play that helps them learn perspective taking [18]. Furthermore, Representational play is assumed to facilitate children to make mental representation, which claimed to contribute to language acquisition [19] and therefore, abstraction. Mental representation is said to determine one's ability to grasp abstract information or tasks. In the visually impaired individuals, mental representation is predicted to be limited, due to the absence of visual experience. This may explain the delay in abstract conceptual formation that leads to difficulty in abstract reasoning. Therefore, the discussion in this study is framed to explain the mechanism between experience, representation, and the reasoning or abstraction.

\section{METHOD}

\section{A. Participants}

Participants were 4 teachers with visual impairment (VI) of special education school for students with visual impairment. The participants have various VI conditions, where 2 teachers were congenitally blind, 1 teacher had a very limited visual 
experience from childhood, and 1 teacher with severe low vision (can see within 20-centimeter span). The youngest were 31 years old, was one with very limited visual experience where he gradually loses sight in his childhood. One congenitally blind teacher was 65 years old, who was actually retired but still teaching for personal reason. The other two teachers were around 45 and 44 years old. Other than the youngest teacher, the participants have more than 15 years teaching experience. These participants were experts, in terms of experience in teaching students with VI, and also supported with personal experience as person with VI.

\section{B. The Data Collecting Method}

The data collection technique in this study is focused group discussion (FGD), interview, and think-aloud protocol. Interview is conducted using semi-structured interview, in which the list of questions is purposefully designed based on the indicators derived from verbal vocabularies and verbal reasoning aspects. The questions were design to be reflective for participants to explore their cognitive process and yet, were aimed to be directive at the same time.

For example, for context generalization the lead question given was "how did you learn the first time about the various different context use of word? For instance, how do you understand different meaning of the word 'Bapak' (translation; a word that could mean 'father' but could also serve as common respectful way to refer to older person) as a way to call your father, and 'Bapak' as a common title as in 'bapakbapak dan ibu-ibu' (translation; 'ladies and gentlemen')".

As in verbal reasoning, the example of the lead question given was "did you find any difficulties in conversation where it involves more than one person in terms of taking turn or when to start and stop talking?" Unplanned additional questions for probing and clarification were often used.

A think-aloud protocol was conducted using a story about a crisis situation. This technique requires participant to give verbal report of their thinking either concurrently, produced when faced with the task, or retrospectively, produced immediately after finishing a task [20]. It is also suggested that concurrent think-aloud provides more explicit, informative, and reliable information about one cognitive process [21]. The protocol was treated as data collecting method based on the assumption that 'people can only report what they are aware of' therefore, think-aloud is argued to have minimum impact on learner's cognitive process [22]. The technique used in this study was concurrent, and used to collect information about learner's cognitive process in solving the question or tasks from the instructor. The think-aloud did not resulting as expected since the nature of the story were beyond the experience they could grasp. The think-aloud resulting from the participants' reflection on their difficulties in meaning making, for instance the word 'traffic', 'ambulance', and some location that were mention in the story. The responses however, were still valuable to understand the verbal reasoning of participants.

The FGD was conducted as a way to gather more comprehensive information where the participants were allowed to compare information from one another. There were
4 groups consist of 4 to 5 people each on the day the data was collected, but this particular article is focusing to only one group of teachers with visual impairment, aiming to discuss how they experience verbal reasoning. The FGD allows them to reflect their own experience from their peers and therefore, provide richer information for the study. The interview and the think-aloud technique were conducted during the FGD so that each of the participant may add up to one another. It is acknowledged however, that it may compromise the depth of one's think-aloud since it was never really focused on one person, but rather a sum of episodes of reflection of each participant.

\section{Data Analysis}

The data collected were verbal reports derived from FGD, interview, and think-aloud. Field notes were also used based on the observation during the tasks performance. The notes from observation were then consulted to the participants and being reviewed and revise according to participants' feedback. Therefore, observation is not considered as the main collecting data technique. The data is analyzed using evident-based method [23]. Evident-based in this study is qualitatively achieved based on the participants' verbal report which based on the indicators already listed. Evidence in this study is following the suggestion of [24] that is "... an observation, fact, of organized body of information offered to support or justify inferences of beliefs in the demonstration of some proposition or matter at issue" [p.27-28]. Therefore, it is very important that the data collector carefully explore the participant's response until the necessary evidence is exposed. In doing so, the interviewer should avoid leading the participants to the intended response.

\section{RESULTS AND DISCUSSION}

For reasoning, participants were listening to a story about a traffic jam that happens in a particular location (e.g. Taman Pelangi). There were some vehicles in the traffic, such as ambulance, fire fighter truck, and hearse. The participants were then told to express their reasoning process in deciding which one goes first and why. This task seemed to be beyond their understandings; however, the participants reflect what they consider affect reasoning for children with VI.

Another story was given that titled 'Jatuh Basah Kuyub' (Falling Soaking Wet). The story was about a little girl name Siti, who lives in a village with his older brother name Didik. One day, his father gave them a horse. They were really happy. On one occasion, Didik was on it and the horse went frightened and ran. Poor Didik fell to a lump of mud. Siti was laughing hard watching it. Didik was full of mud from top to toe. The story was given in Bahasa Indonesia with less complex grammar in terms of time-bind verbs.

According to the participant, the second story can be better understood, because of the concreteness and simplicity of the story.

The participants agreed that learning the meaning of word(s) is very much affected by the context the word first introduced. Parents, who are over protective to their blind child, may result in children not being able to do things 
independently. It may reduce their ability to grasp the 'concrete' and the 'abstract', since the child was being served and lost chance to explore, which normally use to learn the nature of objects. Difficulties are encountered in meaning making for abstract words, such as 'traffic jam', 'function', and 'impact', and even 'running'. This ability, according to the participant, is very much affected by the early stimulation of their parents. Therefore, information from parents is vital to understand the development of reasoning.

One of the participants with limited visual experience remembered visual image of numbers in telephone's buttons. He claimed that the visual image appears each time he thinks of numbers and have helped him to understand the concept of numbers and how it 'operates', for instance, bigger value compared to smaller value based on the number sequence on the 'telephone' imagery that he envisions. This seemed to help him tackling number reasoning which is one among difficult tasks for non-visual individuals. Individuals without vision understands object and often find troubles understanding concepts or symbols. For instance, asking them to remember a 'phone number' is more make sense to them compared to remembering serial of numbers; 'serial of numbers' is therefore, an abstract concept and harder to grasp. Thus, according to the participants, turning an abstract concept into an object is a useful strategy in exploring their actual ability.

On another task, participants were asked to collect information about an object using their tactile sensory. The object is purposefully chosen, because it has unique sign on each side (a piece of 3-dimension puzzle). With tactile sensory participants were then taught how to turn and roll the object on their palm, so that each side with unique sign may rotate and relocated according to the instruction given. The participants were able to perform the task, although the steps of instruction require help from assistance since it has to be practice one by one for them to understand it. It was also suggested to avoid instruction such as '90 degree'; 'right'; and 'left' to give direction. According to the participants, tactile information is preferable rather than verbal instruction. This type of task is easier to understand compared to the former.

Participants suggest that the most important thing in cognition development is the verbal vocabulary, since it is related to the many other aspects in their cognition. Three participants emphasizing on verbal vocabulary and verbal reasoning, in fact, they were suggesting that this may indicate the child's intelligence. Understanding children's verbal ability will also help us to predict the child's general cognitive ability. Again, the participants were emphasizing the effect of parents' teaching. In understanding the use of word in different context and the meaning making by individuals with VI is very much determined by the first time they learn the particular word. If parent did not provide them with proper explanation, it may lead to misconception and will be very difficult to change in the future. Children with VI also learn better with functional explanation rather than word-object association. For instance, teaching an object is called 'spoon' is better understood when it is told during meal time along with an explanation of its function, instead of teaching it as object recognition and say 'this is spoon' and ask the child to remember it using their tactile sensory.
According to the participants, generally children are able to engage effectively in one-on-one conversation, or even with more people. Difficulties may emerge when children are in public areas to recognize who is talking to whom. For example, they may make mistake thinking that someone is talking to them when they are not. This is rather difficult to teach because it requires vision, but basically they able to predict when someone is about to stop talking or is expecting their response which help them as a cue to take turn in conversation. Teaching 'you', 'me', should be taught in condition of two people facing each other and taught by asking them to make physical contact with the other party. Therefore, teaching the word 'we', 'us', 'them', is very difficult and better to be avoided when talking to children with VI. Using consistent word is also helpful for their understanding.

According to [12] reasoning is an ability to choose relevant information and ignore unimportant ones to make inferences beyond the information available. It is a general definition on reasoning, which assumed to apply to all individuals. It is assumed that individual with visual impairment would have different cognitive process due to the absence of visual experience [5], and thus, it is also expected that the reasoning may take different form. The different form of reasoning may be related to the relational reasoning [13] which suggesting that reasoning may not necessarily activate the visual association area in the brain unless the information is visually acquired. The data as reported by the participants in this study suggest that one should trace back the formation of knowledge and concept, which also supported by the finding in [13]. The participants in this study suggest that the representation of information is very important for individual to make inferences. This foundation for this discussion can be separated into two categories, that is the formation of concrete and abstract concepts $[14,25]$. According to [14] the formation of concrete concepts is assumed to be related with the sensory and motor processes and although further clarification is needed, this may also apply to the formation of abstract concepts. The latter though, is assumed to involved more complex process that includes emotional and determined by verbal-symbolic codes which eliminate the visual codes [14]. This aligns to the participants' reflection that stating that vocabulary and verbal reasoning is very crucial to understand individual's cognitive process. For abstract concepts, individual with VI depends on verbal-symbolic codes, in a way it allows them to remember the concept or word although they may find it hard to comprehend the semantic meaning. Some studies use verbalism to refer to such phenomena [26], that is the use of word without a proper understanding of its meaning. [25] suggested though, that congenitally blind individuals may grasp metaphorical expression by using analogy to experiential episodes. Although sometimes inaccurate, blind individuals may make sense conceptual metaphors if the concept can be translated into concrete or touch-related experiences [25].

It can be inferred from the result of the study that the participants often explain their cognition functioning using concrete versus abstract dichotomy. The concrete is often referred to something they can easily experience without visual modality, while abstract refers to concepts that require vision to be understood. Therefore, blind people may understand 
'falling', 'soaking wet', but struggling to understand 'traffic jam', or name of location since it may not be related to any of their personal experience. This seems to align with the participants' reflection that parents who tend to facilitate their children, hence leaving nothing to do for the child, may result in less experience children. Studies seem to support this assumption [10]. This may predict the child's difficulty in grasping many abstract concepts, since it requires them to have something to compare with. In short, blind people is suggested to optimize their sensory-motor modality to acquire abstraction [13], including in translating concept metaphor [25].

Ways to represent information or objects is an important aspect in understanding cognitive ability in VI. On person with VI, spatial orientation is one modality to do so. Furthermore, the ability is not merely about recognizing spatial location or places of things, but also affect one's perspective taking [27]. As mention earlier, spatial orientation may not necessarily relate to visual experience $[13,14,25]$. The participant in this study finds that spatial task is more relatable to their condition. All participants were trained in using tactile sensory to infer information. They in fact prefer information or instruction to be given in demonstration that is accessible using tactile sensory compared to verbal instruction. In this study, spatial orientation is defined as the ability in manipulating, transforming, and integrating information based on the level of difficulty or object complexity ( 2 or 3 dimension, moving or not moving, and number of information/object involved). This definition is the summary from $[7,28]$. From the result of this study, it can be seen that the tactile sensory becomes one among the most important modality (other than hearing) to collect perceptual information. There is however, problem in using this modality which requires specific training, for instance reading braille, and very much affected by the early stimulation and teaching. Using tactile sensory also takes longer time compared to verbal instructions.

As suggested earlier, spatial representation may not require visual experience since there is a strong indication that the cognitive process of spatial and visual perception involves different brain area [14]. One study tried to explore this assumption and found that the representation one uses is more related to the verbal relation [13], that is visuospatial relations, visual relations, spatial relations, and control relations. Each of this type of verbal relation refer to the use of visual or/and spatial representation of object. As for control relation refers to the type of verbal relations that is difficult to be represented both visually and spatially. According to [13] visual images only active when visual representation is involved, in another word, it is not an important thing for reasoning to happen. It also suggests that how one represents an object or information will mostly affect how the reasoning works.

An interesting finding from [16] shows that giving students with VI a chance to explore the nature by climbing, collecting fossils, exploring a bog, with some guided from the expert in the field, help students to collect new understanding about science and help grow their scientific responses. The research was aimed to understand how children with VI adopt geologic conceptual understanding. The study finds that some inaccurate understanding remains after the treatment, but resulting in 'unique misconception' about science that is not presence in their sighted peers [16]. This 'unique misconception' has been noted by several previous studies [10]. The length of the treatment was assumed to be the primary failure in fixing those misconceptions, but further collaboration with formal education in school is predicted to be able to change that misconception. In other word, the treatment of the study may have provided students with ways to represent information or problem in various ways than what they usually do. This is predicted to help them in building scientific understanding. This finding is align to [10] that concept formation in children with VI depends entirely on learning opportunities and experience.

\section{CONCLUSION}

Early experience and stimulation in childhood is very crucial for one's ability to grasp concept formation. Exploration that allows children to collect information from various 'perspective' or sensory modality may help advance the mental representation of objects or information. Abstract representation on visually impaired individuals is not always accurate, but it facilitates individuals to be successful in higher order cognitive tasks, such as abstract reasoning. Further investigation on the link between early experience, mental representation, and concept formation on subjects with visual impairment is suggested for future research.

\section{ACKNOWLEDGEMENT}

The authors are very grateful for the funding from Universitas Negeri Surabaya that makes this study possible. The authors would also like to dedicate special appreciation to Dina Nastiti, a child psychologist who gives valuable contribution in the journey of this research.

\section{REFERENCES}

[1] J. Durando, "Students With visual Impairment or Deaf-Blindness," Common Core State Standards and the Speech-Language Pathologist: Standards-Based Intervention for Special Populations, p. 137, 2015.

[2] L. A. Gunaratne, "Visual impairment: Its effect on cognitive development and behaviour," ed: Intellectual Disability and Health. University of Hertfordshire, 2002.

[3] B. W. Smits and M. J. Mommers, "Differences between blind and sighted children on WISC verbal subtests," New Outlook for the Blind, 1976

[4] M. J. Tobin and E. W. Hill, "Issues in the educational, psychological assessment of visually impaired children: Test-retest reliability of the Williams Intelligence Test for Children with Defective Vision," British Journal of Visual Impairment, vol. 29, pp. 208-214, 2011.

[5] Z. Cattaneo, T. Vecchi, C. Cornoldi, I. Mammarella, D. Bonino, E. Ricciardi, et al., "Imagery and spatial processes in blindness and visual impairment," Neuroscience \& Biobehavioral Reviews, vol. 32, pp. 13461360, 2008.

[6] A. F. Eardley and L. Pring, "Spatial processing, mental imagery, and creativity in individuals with and without sight," European Journal of Cognitive Psychology, vol. 19, pp. 37-58, 2007.

[7] T. Vecchi, C. Tinti, and C. Cornoldi, "Spatial memory and integration processes in congenital blindness," Neuroreport, vol. 15, pp. 2787-2790, 2004

[8] A. Withagen, A. M. L. Kappers, M. P. J. Vervloed, H. Knoors, and L. Verhoeven, "Short term memory and working memory in blind versus sighted children," Research in developmental disabilities, vol. 34, pp. 2161-2172, 2013. 
[9] L. Pring, "Psychological characteristics of children with visual impairments: Learning, memory and imagery," British Journal of Visual Impairment, vol. 26, pp. 159-169, 2008.

[10] K. Jaworska-Biskup, "The world without sight. A comparative study of concept understanding in Polish congenitally totally blind and sighted children," Psychology of language and communication, vol. 15, pp. 27 48, 2011.

[11] R. L. Solso, M. K. MacLin, and O. H. MacLin, Cognitive psychology: Allyn and Bacon Boston, MA, 1998

[12] D. F. Lohman and J. M. Lakin, "Reasoning and intelligence," Handbook of intelligence, pp. 1-47, 2009.

[13] M. Knauff, T. Fangmeier, C. C. Ruff, and P. Johnson-Laird, "Reasoning, models, and images: Behavioral measures and cortical activity," Journal of cognitive neuroscience, vol. 15, pp. 559-573, 2003.

[14] M. Kiefer and F. Pulvermüller, "Conceptual representations in mind and brain: theoretical developments, current evidence and future directions," cortex, vol. 48, pp. 805-825, 2012.

[15] K. C. MacCluskie, R. H. Tunick, J. G. Dial, and D. S. Paul, "The role of vision in the development of abstraction ability," Journal of Visual Impairment \& Blindness, vol. 92, pp. 189-199, 1998.

[16] T. A. Wild, M. P. Hilson, and K. M. Farrand, "Conceptual understanding of geological concepts by students with visual impairements," Journal of Geoscience Education, vol. 61, pp. 222-230, 2013.

[17] T. A. Wild and K. C. Trundle, "Conceptual understandings of seasonal change by middle school students with visual impairments," Journal of Visual Impairment \& Blindness, vol. 104, pp. 107-118, 2010.

[18] E. S. Andersen, A. Dunlea, and L. Kekelis, "The impact of input: Language acquisition in the visually impaired," First language, vol. 13, pp. 23-49, 1993
[19] L. McCune, "A normative study of representational play in the transition to language," Developmental psychology, vol. 31, p. 198, 1995

[20] K. A. Ericsson and H. A. Simon, "Verbal reports as data," Psychological review, vol. 87, p. 215, 1980.

[21] A. L. Brown and J. D. Day, "Macrorules for summarizing texts: The development of expertise," Journal of verbal learning and verbal behavior, vol. 22, pp. 1-14, 1983.

[22] K. A. Ericsson, "12 Capturing Expert Thought with Protocol Analysis: Concurrent Verbalizations of Thinking during Experts' Performance on Representative Tasks," The Cambridge Handbook of Expertise and Expert Performance, p. 192, 2018.

[23] S. L. Odom, E. Brantlinger, R. Gersten, R. H. Horner, B. Thompson, and K. R. Harris, "Research in special education: Scientific methods and evidence-based practices," Exceptional children, vol. 71, pp. 137-148, 2005

[24] J. M. Morse, J. Swanson, and A. J. Kuzel, The nature of qualitative evidence: Sage, 2001

[25] R. A. Minervino, A. Martín, L. M. Tavernini, and M. Trench, "The Understanding of Visual Metaphors by the Congenitally Blind," Frontiers in psychology, vol. 9, 2018.

[26] R. Ranganathan, "A Study On Verbalism Among Visually Impaired Children In Andhra Pradesh," The European Journal of social \& behavioural sciences, vol. 8, p. 1258, 2014.

[27] E. Koustriava and K. Papadopoulos, "Mental rotation ability of individuals with visual impairments," Journal of Visual Impairment \& Blindness, vol. 104, pp. 570-575, 2010.

[28] R. Colom, M. J. Contreras, J. Botella, and J. Santacreu, "Vehicles of spatial ability," Personality and Individual Differences, vol. 32, pp. 903912, 2002. 